Draft VERSiOn MAY 28, 2020

Preprint typeset using $\mathrm{LAT}_{\mathrm{E}} \mathrm{X}$ style emulateapj v. 12/16/11

\title{
IRRADIATED OCEAN PLANETS BRIDGE SUPER-EARTH AND SUB-NEPTUNE POPULATIONS
}

Olivier Mousis, Magali Deleuil, Artyom Aguichine

Aix Marseille Univ, CNRS, CNES, LAM, Marseille, France

EMMANUEL MARCQ

LATMOS/CNRS/Sorbonne Université/UVSQ, 11 boulevard d'Alembert, Guyancourt, F-78280, France

JOSEPH NAAR

Aix Marseille Univ, CNRS, CNES, LAM, Marseille, France and

LATMOS/CNRS/Sorbonne Université/UVSQ, 11 boulevard d'Alembert, Guyancourt, F-78280, France

Lorena ACUÑa Aguirre

Aix Marseille Univ, CNRS, CNES, LAM, Marseille, France

BASTIEN BRUGGER

Department of Astronomy, Cornell University, Ithaca, NY 14853, USA

Thomas Gonçalves

Aix Marseille Univ, CNRS, CNES, LAM, Marseille, France

(Received; Revised; Accepted May 28, 2020)

Draft version May 28, 2020

\section{ABSTRACT}

Small planets $\left(\sim 1-3.9 R_{\oplus}\right)$ constitute more than half of the inventory of the 4000-plus exoplanets discovered so far. Smaller planets are sufficiently dense to be rocky, but those with radii larger than $\sim 1.6 R_{\oplus}$ are thought to display in many cases hydrogen/helium gaseous envelopes up to $\sim 30 \%$ of the planetary mass. These low-mass planets are highly irradiated and the question of their origin, evolution, and possible links remains open. Here we show that close-in ocean planets affected by greenhouse effect display hydrospheres in supercritical state, which generate inflated atmospheres without invoking the presence of large hydrogen/helium gaseous envelopes. We present a new set of mass-radius relationships for ocean planets with different compositions and different equilibrium temperatures, which are found to be well adapted to low-density sub-Neptune planets. Our model suggests that super-Earths and water-rich sub-Neptunes could belong to the same family of planets, i.e. hydrogen/helium-free planets, with differences between their interiors simply resulting from the variation in the water content.

Subject headings: planets and satellites: composition, planets and satellites: ocean, planets and satellites: atmospheres, planets and satellites: detection

\section{INTRODUCTION}

With radii ranging between those of the Earth $\left(1 R_{\oplus}\right)$ and Neptune $\left(\sim 3.9 R_{\oplus}\right)$, small planets constitute more than half of the inventory of the 4000-plus exoplanets discovered so far ${ }^{1}$. Smaller planets are sufficiently dense to be rocky, but those with radii larger than $\sim 1.6 R_{\oplus}$ are thought to display large amounts of volatiles, including in many cases hydrogen/helium gaseous envelopes up to $\sim 30 \%$ of the planetary mass (Lopez et al. 2012; Weiss \& Marcy 2014, Rogers 2015). With orbital periods less than 100 days, these low-mass planets are highly irradiated and their origin, evolution, and possible links are still debated (Venturini \& Helled 2017; Dorn et al. 2017; Jin \& Mordasini 2018, Zeng et al. 2019).

The composition of the small planet population is often assessed by comparing their mass and radius measure-

olivier.mousis@lam.fr

${ }_{1}^{1}$ https://exoplanetarchive.ipac.caltech.edu ments, when available, to theoretical mass/radius relationships. While super-Earths appear compatible with more or less differentiated rocky planets, sub-Neptunes fall near non physical curves of planets composed of pure water, suggesting instead a solid core surrounded by a hydrogen/helium gaseous envelope up to $\sim 30 \%$ of the planetary mass (Fulton et al. 2017; Fulton \& Petigura 2018. Lopez et al. 2012; Rogers 2015; Venturini \& Helled 2017; Weiss \& Marcy 2014; Dorn et al. |2017; Jin \& Mordasini 2018: Zeng et al. 2019). However, even if the existence of sub-Neptunes with low metallicities (i.e. with hydrogenrich atmospheres) is supported by spectroscopic observations (Benneke et al. 2019a), $\mathrm{H}_{2} \mathrm{O}$ absorption bands have also been detected in the exo-Neptunes HAT-P-26b (MacDonald \& Madhusudhan 2019) and K2-18b (Benneke et al. 2019b).

There is no clear consensus on how these small exoplanets populations might have formed. In-situ formation faces number of issues (Ogihara et al. 2015) and mi- 
gration appears as the most likely scenario. In that case, planetary embryos or building blocks might have formed in the cold regions of the protoplanetary disk and migrated inward. As a result of their formation location, they should contain a significant fraction of water ice. This leads to reconsider the possible existence of massive water-rich planets, as initially suggested by Léger et al. (2004). Water-rich worlds (Europa, Titan, Enceladus, Pluto, etc) are ubiquitous in our solar system, and the building blocks of Neptune and Uranus are also supposed to be water rich (Mousis et al. 2018). However none of the existing mass/radius relationships fully explores the physical properties of such ocean planets, i.e. planets displaying a significant fraction of liquid water ( 50 wt \%), especially in the domain of high surface temperature. For instance, Zeng \& Sasselov (2014) and Thomas \& Madhusudhan (2016) pointed out the possible presence of supercritical water in ocean planets, but both were lacking in an atmospheric prescription. On the other hand, a recent investigation of mass-radius relationships for waterrich terrestrial planets (up to $5 \mathrm{wt} \%$ of water) endowed with water-dominated steam atmospheres suggests that such planets present radii larger those of planets with water in liquid or solid phases (Turbet et al. 2020). However, this study needs further reassessment because the water layers of the most hydrated planets considered encompass pressure-temperature ranges corresponding to those of a supercritical fluid, which is not implemented in the used atmospheric model.

Here, to investigate the role of irradiation on ocean planets, we use a combination of two one-dimensional models, i.e. a fully differentiated planet interior model (Brugger et al. 2017) and a steam atmosphere model (Marcq 2012; Marcq et al. 2017) connected at a 1000-bar pressure, both using a supercritical water equations of state (EOS). We show that close-in ocean planets (Léger et al. 2004) affected by greenhouse effect display hydrospheres in supercritical state, which generate inflated atmospheres without invoking the presence of large $\mathrm{H} / \mathrm{He}$ gaseous envelopes. We derive a new set of mass-radius relationships for ocean planets with different compositions and different equilibrium temperatures, well adapted to low-density sub-Neptune planets. While it does not preclude the existence of other hydrogen-helium-rich subNeptunes, our model suggests that super-Earths and water-rich sub-Neptunes could belong to the same family of planets. The differences between their interiors could simply result from the variation of the water content in those planets.

\section{MODEL}

\subsection{Interior model}

We use the internal model of solid planet developed by Brugger et al. (2017). It takes as inputs the planetary mass and chemical composition $(\mathrm{Mg} / \mathrm{Si}$, Fe/Si mole ratios and water mass fraction), and computes the resulting radius and internal structure of the planet (Brugger et al. 2017). The internal structure computes the pressure $P(r)$, the temperature $T(r)$, the gravity acceleration $g(r)$, and the density $\rho(r)$ as a function of radius. These quantities are integrated following an iterative scheme until convergence is reached. Along the radius $r$ of the planet, the pressure $P(r)$ is calculated via different EOS, which are chosen depending on the material that composes the considered layer. The different layers include the core, the lower and upper mantles, the high pressure ice, and a liquid hydrosphere (Brugger et al. 2017). To account for the effects of irradiation, as expected for the close-in population, a water phase in supercritical state has been added to the hydrosphere. For given density and temperature, the supercritical layer pressure is calculated via an EOS obtained from data generated by molecular level computer simulations that consider simple point-charge potential models to which average polarization corrections have been added (Duan \& Zhang 2006). This EOS is written as:

$$
\begin{array}{r}
Z=\frac{P V}{R T}=1+\frac{B V_{c}}{V}+\frac{C V_{c}^{2}}{V^{2}}+\frac{D V_{c}^{4}}{V^{4}}+\frac{E V_{c}^{5}}{V^{5}}+ \\
\frac{F V_{c}^{2}}{V^{2}} \times\left(\beta+\frac{\gamma V_{c}^{2}}{V^{2}}\right) \exp \left(-\frac{\gamma \mathrm{V}_{\mathrm{c}}^{2}}{\mathrm{~V}^{2}}\right),
\end{array}
$$

where $R=83.14467 \mathrm{~cm}^{3} \mathrm{bar} /(\mathrm{K} \mathrm{mol})$ is the universal gas constant. Parameters $B, C, D, E$, and $F$ in Eq. 1 are calculated via the following equations:

$$
\begin{gathered}
B=a_{1}+\frac{a_{2}}{T_{r}^{2}}+\frac{a_{3}}{T_{r}^{3}} \\
C=a_{4}+\frac{a_{5}}{T_{r}^{2}}+\frac{a_{6}}{T_{r}^{3}} \\
D=a_{7}+\frac{a_{8}}{T_{r}^{2}}+\frac{a_{9}}{T_{r}^{3}} \\
E=a_{10}+\frac{a_{11}}{T_{r}^{2}}+\frac{a_{12}}{T_{r}^{3}} \\
F=\frac{\alpha}{T_{r}^{3}} \\
T_{r}=\frac{T}{T_{c}} \\
F=\frac{R T_{c}}{P_{c}}
\end{gathered}
$$

where $T_{c}$ and $P_{c}$ are the critical temperature and critical pressure respectively. Here, $T_{c}=647.25 \mathrm{~K}$, and $P_{c}=$ $221.19 \mathrm{~cm}^{3} / \mathrm{mol}$. Parameters $a_{1}-a_{12}, \alpha, \beta$, and $\gamma$ valid in the $0.2-10$ GPa range are summarized in Table 1 . We refer the reader to the study of Duan \& Zhang (2006) for details.

The resulting EOS (hereafter DZ06) agrees within a $\pm 0.6 \%$ deviation with the well-known IAPWS95 formulation (Wagner \& Pruß2002), which provides an accurate EOS based on experimental data within the $\sim 0-1.0 \mathrm{GPa}$ pressure range. At higher pressure, the DZ06 EOS has been shown to compute the pressure within a $\pm 1.3 \%$ deviation up to $10.0 \mathrm{GPa}$. Above, comparisons with simulated data (Duan et al. 1996, Duan \& Zhang 2006) shows it remains within a $\pm 5.0 \%$ deviation up to $35 \mathrm{GPa}$.

The adiabatic temperature profile within the supercritical layer depends on the Grüneisen parameter, which has a strong dependence with both density and temperature. In the supercritical layer, this parameter is derived from a bilinear interpolation of a grid of data available in the 
TABLE 1

EOS PARAMETERS

\begin{tabular}{lc}
\hline \hline Parameter & Value \\
\hline$a_{1}$ & $4.68071541 \times 10^{-02}$ \\
$a_{2}$ & $-2.81275941 \times 10^{-01}$ \\
$a_{3}$ & $-2.43926365 \times 10^{-01}$ \\
$a_{4}$ & $1.10016958 \times 10^{-02}$ \\
$a_{5}$ & $-3.86603525 \times 10^{-02}$ \\
$a_{6}$ & $9.30095461 \times 10^{-02}$ \\
$a_{7}$ & $-1.15747171 \times 10^{-05}$ \\
$a_{8}$ & $4.19873848 \times 10^{-04}$ \\
$a_{9}$ & $-5.82739501 \times 10^{-04}$ \\
$a_{10}$ & $1.00936000 \times 10^{-06}$ \\
$a_{11}$ & $-1.01713593 \times 10^{-05}$ \\
$a_{12}$ & $1.63934213 \times 10^{-05}$ \\
$\alpha$ & $-4.49505919 \times 10^{-02}$ \\
$\beta$ & $-3.15028174 \times 10^{-01}$ \\
$\gamma$ & $1.25000000 \times 10^{-02}$ \\
\hline
\end{tabular}

python library for IAPWS standard calculation of water and steam properties ${ }^{2}$. This grid gives a range of Grüneisen parameters for temperatures up to $10^{4} \mathrm{~K}$ and supercritical water densities up to $2500 \mathrm{~kg} / \mathrm{m}^{3}$, corresponding to pressures up to $\sim 150 \mathrm{GPa}$, a value exceeding the one at the center of a $20 M_{\oplus}$ planet fully made of water. When deriving this grid, the IAPWS team focused on the behavior of the extrapolation of the analytical formulation, and ensured it remains physically correct in domains of high pressure/temperature, which are relevant to exoplanetary interiors. The Grüneisen parameter's profile is then expected to have a correct physical behavior, albeit with increasing uncertainties when going deeper in the planet. However, we find this to be of secondary importance regarding planetary radius as the Grüneisen parameter is basically a proxy of thermal expansivity along pressure variations, which rapidly becomes of second order when pressure increases.

\subsection{Atmosphere model}

The atmosphere model (Marcq 2012 Marcq et al. 2017) takes over the hydrosphere at water column pressures lower than 1000 bar, where the $\mathrm{H}_{2} \mathrm{O}$ envelope behaves more and more like a hot and dense steam atmosphere as the pressure drops. The used model is based on a $T(P)$ profile prescription (Kasting 1988 ) starting from the 1000-bar level (unsaturated since $T(1000$ bar $\left.)>T_{\text {critical }}\right)$ upwards, assuming a dry adiabat, and switching optionally to a moist adiabat (where $\left.T(P)=T_{\text {saturation }}(P)\right)$ if/when saturation reaches unity. Once the temperature reaches the top temperature, here set to $200 \mathrm{~K}$, an isothermal radiative mesosphere $T=$ $T_{\text {top }}$ is assumed up to the $0.1 \mathrm{~Pa}$ topmost level. Moreover, steam is not treated as an ideal gas, and the EOS is taken instead from the NBS/NRC steam tables (Haar et al. 1984). This enables a smooth transition of the $T(P)$ profile with the interior model at equilibrium temperatures of 300 and $650 \mathrm{~K}$, at least. Altitudes are computed assuming hydrostatic equilibrium. Shortwave and thermal fluxes are then computed using 4-stream approximation. Gaseous (line and continuum) absorptions are computed using the $k$-correlated method. Rayleigh opacity

\footnotetext{
2 https://pypi.org/project/iapws/\#description
}

is also included. $T(1000$ bar $)$ is iteratively chosen so that the thermal flux at the top of the atmosphere is equal to $\sigma T_{\mathrm{eq}}{ }^{4}$. We finally chose the radius/altitude of the 20 mbar level as the observable, transiting radius Grimm et al. 2018).

\section{IMPLICATIONS FOR THE MASS-RADIUS RELATIONSHIPS}

Figure 1 displays the pressure and temperature profiles (hereafter $(P, T)$ profiles) of $1-15 M_{\oplus}$ supercritical ocean planets fully constituted of water with equilibrium temperatures of 300,650 , and $1200 \mathrm{~K}$, superimposed onto the water phase diagram. With the lack of rocky cores, these planets present unphysical interiors but they have the merit to display hydrospheres over a large range of temperatures and pressures. The fluid-ice VII transition law is fitted from data between 3 and $60 \mathrm{GPa}$ (Frank et al. 2004). The other phase-change laws are collected from a compilation of thermodynamic data (Wagner \& Pruß 2002), and from the website of the International Association for the Properties of Water and Steam (IAPWS) The $(P, T)$ profiles expand from the base of the hydrosphere (here the center of the planet) to the top of the $\mathrm{H}_{2} \mathrm{O}$-dominated atmosphere set to $0.1 \mathrm{~Pa}$. Most of the hydrospheres remain in the supercritical regime and those of smallest planets are located well below ice VII, with a fluid-ice VII transition law valid up to $60 \mathrm{GPa}$ (Frank et al. 2004) in the water phase diagram. Beyond this pressure range, the phase change from supercritical to high pressure ices (VII or X) is neglected because the temperature/pressure region remains widely unknown in this region.

\footnotetext{
${ }^{3}$ http://www.iapws.org/relguide/MeltSub.html
} 


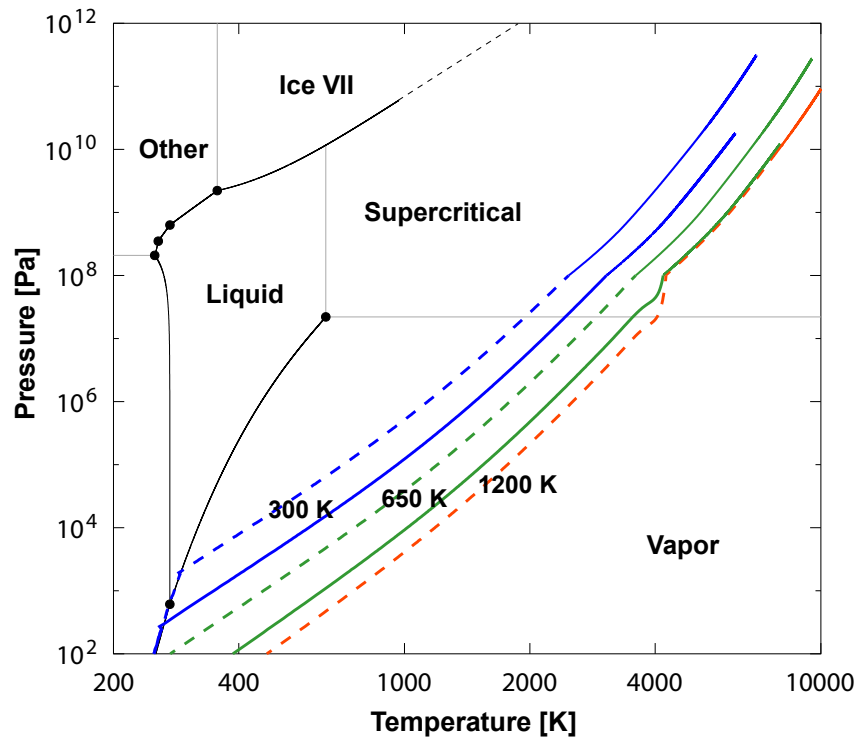

FIG. 1.- Pressure and temperature profiles of hydrospheres of 1 and $15 M_{\oplus}$ supercritical ocean planets fully constituted of water with equilibrium temperatures of 300,650 , and $1200 \mathrm{~K}$ superimposed onto the water phase diagram. Colored solid and dashed curves correspond to 1 and $15 M_{\oplus}$ planets, respectively. The black dashed line delimits a hypothetical transition between the supercritical phase and high pressure ice (see text). Dashed lines change to solid lines when the interior model takes over from the atmosphere model. The $1 M_{\oplus}$ case with an equilibrium temperature of $1200 \mathrm{~K}$ is removed because its atmosphere is hydrostatically unstable (see Fig. 2).

Figure 2 represents the mass/radius relationships of supercritical ocean planets calculated in the $0.6-20 M_{\oplus}$ range for equilibrium temperatures of 300,650 , and 1200 $\mathrm{K}$, corresponding to distances of $0.72,0.15$, and $0.04 \mathrm{AU}$ from a solar-type star, respectively, assuming an Earthlike albedo. We explore different interior compositions, ranging between the two extreme and unrealistic cases of pure core and pure supercritical water (SW) compositions, to compare the effects on the resulting observables. The presence of a thick $\mathrm{H}_{2} \mathrm{O}$-dominated atmosphere generates a strong runaway greenhouse effect causing the presence of a supercritical hydrosphere, even if the equilibrium temperature of the planet is lower than the critical temperature of water $\left(T_{\text {critical }} \sim 650 \mathrm{~K}\right)$. Because the core-mantle boundary is not firmly defined at very high pressure and temperature (Hakim et al. 2018), we assume here the core and mantle form a unique phase constituted of silicate rocks, including bridgmanite $(\mathrm{Mg}, \mathrm{Fe}) \mathrm{SiO}_{3}$, and ferro-periclase, $(\mathrm{Mg}, \mathrm{Fe}) \mathrm{O}$ (Brugger et al. 2017).

Figure 2 shows that some members of the subNeptunes population can be well matched by mass/radius curves corresponding to ocean planets with significant supercritical hydrospheres. For the sake of comparison, the sub-Neptune K2-38b, in which $\mathrm{H}_{2} \mathrm{O}$ absorption bands have been recently observed (Benneke et al. 2019b), is also represented in Fig. 2 If one considers the central values of its measured mass and radius $\left(M_{p}=8.63 M_{\oplus}\right.$ and $R_{p}=2.61 R_{\oplus}$; Benneke et al. $(2019 \mathrm{~b}))$, the composition of this planet cannot be explained via the use of classical super-Earths interior models, and requires an extra amount of hydrogenhelium. On the other hand, it is fully matched by models made by a rocky core-mantle toped by a supercritical
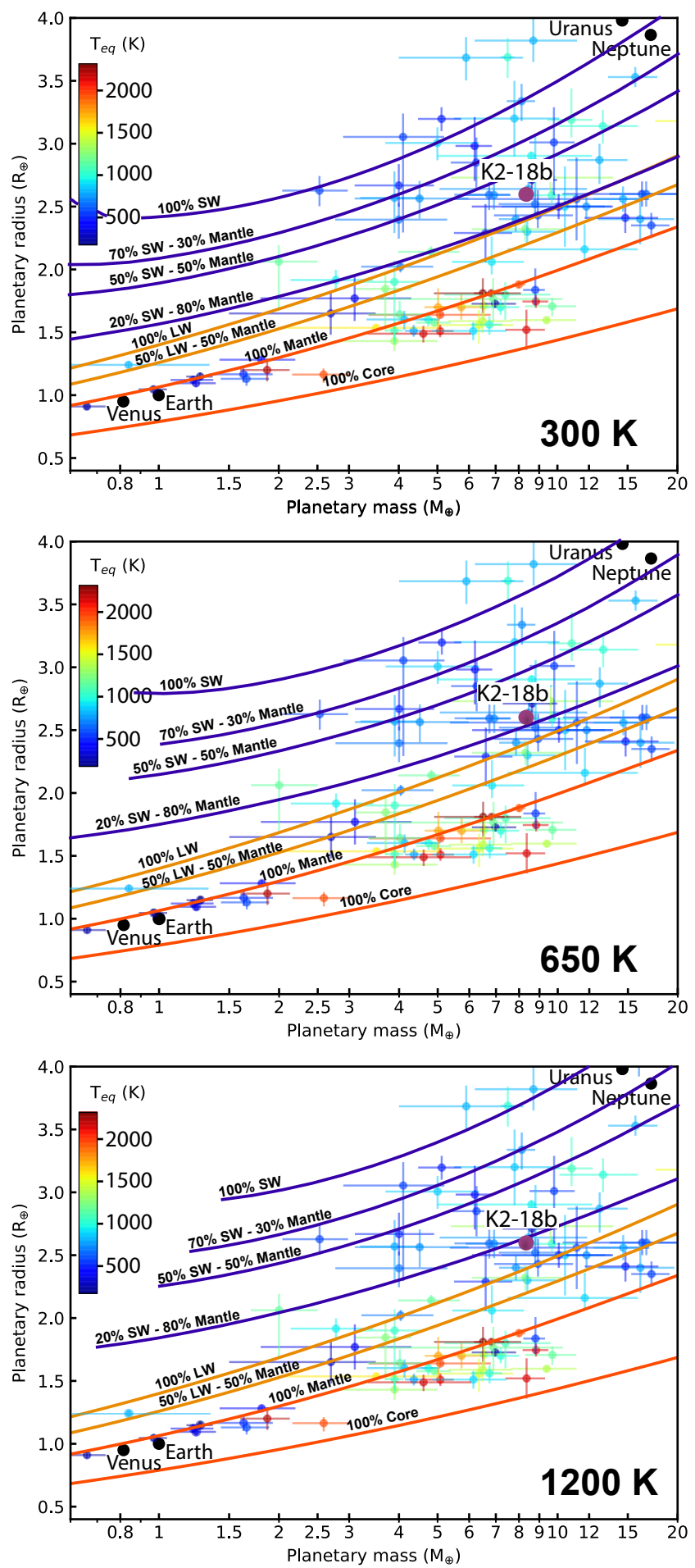

FiG. 2.- Mass-radius diagrams determined for exoplanets with masses in the $0.6-20 M_{\oplus}$ range, and equilibrium temperatures of $300 \mathrm{~K}, 650 \mathrm{~K}$, and $1200 \mathrm{~K}$. Mass-radius curves are calculated for several planetary compositions: $100 \%$ core and $100 \%$ mantle (red curves), liquid water (LW) hydrosphere (brown curves) and supercritical water (SW) hydrosphere (blue curves) topping mantle-like composition interiors. Planetary data are taken from the NASA exoplanet archive and updated to 20th July 2019. Hydrostatically unstable atmospheres (defined when the altitude at $0.1 \mathrm{~Pa}$ tends towards infinity) around the hotter and smaller planets are excluded from the mass-radius relationships.

water layer and a steam atmosphere irradiated at $300 \mathrm{~K}$, 
a value close to the equilibrium temperature estimated to be $\sim 257 \mathrm{~K}$ (Benneke et al. 2019b). In this case, the mass fraction of incorporated water is $\sim 37 \%$, a value in the same range as those derived for the water worlds in our solar system.

\section{DISCUSSION}

Our model explores the assumption of water-rich and supercritical planets as an alternative or a complement to sub-Neptunes with hydrogen/helium gaseous envelopes. This indeed does not exclude the existence of such minigiants but suggests that super-Earths and a fraction of sub-Neptunes could belong to the same family of planets, with differences between their interiors simply resulting from the variation in the water content. Because of the proximity to their host star, for those which are water-rich, the strong insolation associated to runaway greenhouse effect in their atmospheres generates inflated supercritical hydrospheres. As a result, they would exhibit larger radii compared to similar bodies with a very low water content located at higher distances to the star. Both Zeng \& Sasselov (2014) and Thomas \& Madhusudhan (2016) also found that small planets with supercritical water layers could exhibit larger radii than planets of the same masses with solid or liquid hydrospheres. However, these increases were found more moderate than those derived from our model because of the absence of coupling with water-rich atmosphere models. The same effect has been recently reported in the case of irradiated super-Earths displaying water steam atmospheres in contact with rocky mantles (Turbet et al. 2020). However, the outcomes of this study require a new assessment via the implementation of a dedicated supercritical water EOS in the atmosphere model. Meanwhile, our model also presents some limitations. The convergence observed in Fig. 11 at the 1000-bar interface between the pressure-temperature profiles of the $15 M_{\oplus}$ planet at $1200 \mathrm{~K}$ and the $1 M_{\oplus}$ planet at $650 \mathrm{~K}$ corresponds to the use of an EOS for supercritical water in the atmosphere model different from the one employed in the interior model, and which slightly departs from its validity range.

We underline that high pressure ices such as Ice $\mathrm{X}$ and Ice XI (Zeng \& Sasselov 2014) are not taken into account in our model. However, the pressure reached at the base of the hydrosphere of $20 M_{\oplus}$ planets including $50 \%$ water is about $600 \mathrm{GPa}$ with temperatures always greater than $4000 \mathrm{~K}$. This temperature-pressure range, close to the one reached at the center of the $15 M_{\oplus}$ pure water planet $(500 \mathrm{GPa})$ shown in Fig. 1. is out of the stability domain of Ice X and Ice XI (see Fig. 1 of Zeng \& Sasselov (2014)). We therefore estimate that this effect should be marginal up to $20 M_{\oplus}$. Also, high temperatures achieved at the base of the hydrosphere may be sufficient to induce melting of the silicate layer. As a result, much of the volatile content of the planet may actually be dissolved within the liquid silicate mantle (Kite et al. 2019, 2020).

Interestingly, planets possessing exactly the mass and radius of Neptune could be matched by ocean planets if these latter contain $\sim 70 \%$ of supercritical water, de- pending on their orbital distance and the type of their host star. Our model implies that both super-Earths and water-rich sub-Neptunes have grown from building blocks with a wide range of ice-to-rock ratios. This hypothesis is supported by recent dynamical simulations showing that the observed distributions of close-in small planets is matched by the presence of both icerich and dry planets (Izidoro et al. 2017, 2019). Waterrich small planets should have grown from ice-rich building embryos formed beyond the snowline in protoplanetary disks while dry ones would have grown from rocky embryos formed within the snowline. Ice-rich embryos would have subsequently migrated inward the disk, catalyzing the growth of purely rocky planets interior to the ice-rich ones (Raymond et al. 2018a b).

Observations have shown that the population of small planets follows a bimodal distribution peaking at $\sim 1.3 R_{\oplus}$ (super-Earths) and $2.4 R_{\oplus}$ (sub-Neptunes), with few planets in between (Fulton et al. 2017; Fulton \& Petigura 2018). A study, combining planetary interior models based on a mixture of rocks and ices associated with Monte Carlo simulations, has recently addressed this question (Zeng et al. 2019). The authors found that the $2-4 R_{\oplus}$ planets should contain significant amounts of $\mathrm{H}_{2} \mathrm{O}$-dominated ices in addition to rock, with perhaps more than half, by mass. They also derived that planets with radii $>3 R_{\oplus}$ would generally require the presence of a gaseous envelope. The physical assumptions formulated in this study are however questionable since highly irradiated water-rich close-in planets should display supercritical and inflated hydrospheres instead of being simply ice-rich. It has also been proposed that the gap between the super-Earths and sub-Neptunes could be accounted by the evaporation of gaseous envelopes, due to the host star irradiation (Owen \& Murray-Clay 2018; Jin \& Mordasini 2018). The same process could also happen to water-rich sub-Neptunes displaying supercritical envelopes: studies of water loss from terrestrial planets orbiting ultracool dwarfs assume that photolysis, i.e. the conversion of $\mathrm{H}_{2} \mathrm{O}$ steam into $\mathrm{H}_{2}$ gas, is not a limiting process in the escape mechanism (Bolmont et al. 2017). The same assumption would remain valid in the case of stars displaying stronger FUV fluxes.

In summary, models of water-rich planets with supercritical envelopes can explain the observed physical properties of some of the planets among the small planet population. They offer an interesting alternative to current dichotomy between rocky and gaseous-rich planets. This quantitative exploration of the role of supercritical water in planetary envelopes, which underlines the importance of composition in case of strong irradiation, will be extended by the development of a model describing the planets' interior and atmosphere in a more consistent way. This work also highlights the need for improved EOS in the high pressure and high temperature regime of the water phase diagram.

O.M. and M.D. acknowledge support from CNES. We acknowledge an anonymous Referee whose comments helped improve and clarify this manuscript.

\section{REFERENCES}


Bolmont, E., Selsis, F., Owen, J. E., et al. 2017, MNRAS, 464, 3728

Brugger, B., Mousis, O., Deleuil, M., et al. 2017, ApJ, 850, 93

Dorn, C., Venturini, J., Khan, A., et al. 2017, A\&A, 597, A37

Duan, Z., \& Zhang, Z. 2006, Geochim. Cosmochim. Acta, 70, 2311

Duan, Z., Møller, N., \& Weare, J. H. 1996,

Geochim. Cosmochim. Acta, 60, 1209

Grimm, S. L., Demory, B.-O., Gillon, M., et al. 2018, A\&A, 613, A68

Frank, M. R., Fei, Y., \& Hu, J. 2004, Geochim. Cosmochim. Acta, 68, 2781

Fulton, B. J., \& Petigura, E. A. 2018, AJ, 156, 264

Fulton, B. J., Petigura, E. A., Howard, A. W., et al. 2017, AJ, 154, 109

Haar, L., Gallagher, J., Kell, G., \& National Standard Reference Data System (U.S.). NBS/NRC Steam Tables: Thermodynamic and Transport Properties and Computer Programs for Vapor and Liquid States of Water in SI Units, Hemisphere Publ. Corp., Washington, D. C. 1984

Hakim, K., Rivoldini, A., Van Hoolst, T., et al. 2018, Icarus, 313, 61

Izidoro, A., Bitsch, B., Raymond, S. N., et al. 2019, arXiv e-prints, arXiv:1902.08772

Izidoro, A., Ogihara, M., Raymond, S. N., et al. 2017, MNRAS, 470, 1750

Jin, S., \& Mordasini, C. 2018, ApJ, 853, 163

Kasting, J. F. 1988, Icarus, 74, 472

Kite, E. S., Fegley, B., Schaefer, L., et al. 2020, ApJ, 891, 111

Kite, E. S., Fegley, B., Schaefer, L., et al. 2019, ApJ, 887, L33
Léger, A., Selsis, F., Sotin, C., et al. 2004, Icarus, 169, 499 Lopez, E. D., Fortney, J. J., \& Miller, N. 2012, ApJ, 761, 59 MacDonald, R. J., \& Madhusudhan, N. 2019, MNRAS, 486, 1292 Marcq, E., Salvador, A., Massol, H., et al. 2017, Journal of Geophysical Research (Planets), 122, 1539

Marcq, E. 2012, Journal of Geophysical Research (Planets), 117, E01001

Mousis, O., Atkinson, D. H., Cavalié, T., et al. 2018, Planet. Space Sci., 155, 12

Ogihara, M., Morbidelli, A., \& Guillot, T. 2015, A\&A, 578, A36

Owen, J. E., \& Murray-Clay, R. 2018, MNRAS, 480, 2206

Raymond, S. N., Izidoro, A., \& Morbidelli, A. 2018b, arXiv e-prints, arXiv:1812.01033

Raymond, S. N., Boulet, T., Izidoro, A., et al. 2018a, MNRAS, 479, L81

Rogers, L. A. 2015, ApJ, 801, 41

Thomas, S. W., \& Madhusudhan, N. 2016, MNRAS, 458, 1330

Turbet, M., Bolmont, E., Ehrenreich, D., et al. 2020, https://doi.org/10.1051/0004-6361/201937151

Venturini, J., \& Helled, R. 2017, ApJ, 848, 95

Wagner, W., \& Pruß, A. 2002, Journal of Physical and Chemical Reference Data, 31, 387

Weiss, L. M., \& Marcy, G. W. 2014, ApJ, 783, L6

Zeng, L., Jacobsen, S. B., Sasselov, D. D., et al. 2019,

Proceedings of the National Academy of Science, 116, 9723

Zeng, L., \& Sasselov, D. 2014, ApJ, 784, 96 